$\mathcal{O S}_{\text {doi: } 10.3765 / \text { sp.2.4a }}$

\title{
Background materials for "Embedded implicatures?!?"
}

Bart Geurts
Nausicaa Pouscoulous

In the following, we list the instructions and materials that weren't given in the paper.

\section{Experiments 1a-b: Instructions}

Dans chacune des pages suivantes un personnage énonce une phrase. Votre tâche est de décider si l'on peut déduire de ce qu'il a dit la proposition qui suit. Si vous pensez que l'on peut, cochez la case "Oui”. Sinon, cochez la case "Non".

Par exemple:

Emilie a dit : “Christian est retourné voir Le Seigneur des Anneaux."

En déduiriez-vous que Christian est déjà allé voir Le Seigneur des Anneaux?

$\square$ Oui $\square$ Non

Dans ce cas la réponse correcte est “Oui”.

Ne passez pas trop de temps sur chaque page, mais ne vous précipitez pas non plus. Choisissez simplement la première réponse qui vous vient à l'esprit. Répondez à une question à la fois et ne revenez pas en arrière une fois que vous avez tourné la page.

\section{Experiments 1a-b: Materials}

\section{Complex sentences with "think"}

- "Betty pense que Fred a écouté certaines des chansons du dernier album de Madonna.” En déduiriez-vous que Betty exclut que Fred ait écouté toutes les chansons du dernier album de Madonna?

- "Betty pense que Fred a écouté certaines des symphonies de Beethoven.” En déduiriez-vous que Betty exclut que Fred ait écouté toutes les symphonies de Beethoven? 
- "Betty pense que Fred a écouté certains des albums de Renaud." En déduiriezvous que Betty exclut que Fred ait écouté tous les albums de Renaud?

- "Betty pense que Fred a écouté certains des opéras de Verdi." En déduiriezvous que Betty exclut que Fred ait écouté tous les opéras de Verdi?

- "Betty pense que Fred a acheté certaines des Harry pièces de Racine.” En déduiriez-vous que Betty exclut que Fred ait acheté toutes les pièces de Racine?

- "Betty pense que Fred a acheté certaines des symphonies de Beethoven." En déduiriez-vous que Betty exclut que Fred ait acheté toutes les symphonies de Beethoven?

- "Betty pense que Fred a acheté certains des albums de Renaud." En déduiriezvous que Betty exclut que Fred ait acheté tous les albums de Renaud?

- "Betty pense que Fred a acheté certains des Alien." En déduiriez-vous que Betty exclut que Fred ait acheté tous les Alien?

- "Betty pense que Fred a acheté certains des DVD de Harry Potter." En déduiriez-vous que Betty exclut que Fred ait acheté tous les DVD de Harry Potter?

- "Betty pense que Fred a acheté certains des films de Tarantino." En déduiriezvous que Betty exclut que Fred ait acheté tous les films de Tarantino?

- "Betty pense que Fred a acheté certains des Harry Potter." En déduiriez-vous que Betty exclut que Fred ait acheté tous les Harry Potter?

- "Betty pense que Fred a acheté certains des James Bond." En déduiriez-vous que Betty exclut que Fred ait acheté tous les James Bond?

- "Betty pense que Fred a acheté certains des opéras de Verdi." En déduiriezvous que Betty exclut que Fred ait acheté tous les opéras de Verdi?

- "Betty pense que Fred a acheté certains des romans de Daniel Pennac." En déduiriez-vous que Betty exclut que Fred ait acheté tous les romans de Daniel Pennac?

- "Betty pense que Fred a acheté certains des romans de Flaubert." En déduiriezvous que Betty exclut que Fred ait acheté tous les romans de Flaubert?

- "Betty pense que Fred a loué certaines des saisons de Sex in the City." En déduiriez-vous que Betty exclut que Fred ait loué toutes les saisons de Sex in the City?

- "Betty pense que Fred a loué certains des Alien." En déduiriez-vous que Betty exclut que Fred ait loué tous les Alien?

- "Betty pense que Fred a loué certains des DVD de Harry Potter." En déduiriezvous que Betty exclut que Fred ait loué tous les DVD de Harry Potter?

- "Betty pense que Fred a loué certains des films de Tarantino." En déduiriez- 
Background for "Embedded implicatures?!?"

vous que Betty exclut que Fred ait loué tous les films de Tarantino?

- "Betty pense que Fred a loué certains des James Bond." En déduiriez-vous que Betty exclut que Fred ait loué tous les James Bond?

- "Betty pense que Fred a lu certaines des Harry pièces de Racine." En déduiriezvous que Betty exclut que Fred ait lu toutes les pièces de Racine?

- "Betty pense que Fred a lu certains des Harry Potter." En déduiriez-vous que Betty exclut que Fred ait lu tous les Harry Potter?

- "Betty pense que Fred a lu certains des poèmes des Fleurs du Mal." En déduiriez-vous que Betty exclut que Fred ait lu tous les poèmes des Fleurs du Mal?

- "Betty pense que Fred a lu certains des romans de Daniel Pennac." En déduiriez-vous que Betty exclut que Fred ait lu tous les romans de Daniel Pennac?

- "Betty pense que Fred a lu certains des romans de Flaubert." En déduiriezvous que Betty exclut que Fred ait lu tous les romans de Flaubert?

- "Betty pense que Fred a vu certaines des saisons de Sex in the City." En déduiriez-vous que Betty exclut que Fred ait vu toutes les saisons de Sex in the City?

- "Betty pense que Fred a vu certains des Alien." En déduiriez-vous que Betty exclut que Fred ait vu tous les Alien?

- "Betty pense que Fred a vu certains des films de Tarantino." En déduiriez-vous que Betty exclut que Fred ait vu tous les films de Tarantino?

- "Betty pense que Fred a vu certains des Harry Potter." En déduiriez-vous que Betty exclut que Fred ait vu tous les Harry Potter?

- "Betty pense que Fred a vu certains des James Bond." En déduiriez-vous que Betty exclut que Fred ait vu tous les James Bond?

\section{Complex sentences with "want"}

"Betty veut que Fred écoute certaines des chansons du dernier album de Madonna." En déduiriez-vous que Betty veut que Fred n'écoute pas toutes les chansons du dernier album de Madonna?

- "Betty veut que Fred écoute certaines des symphonies de Beethoven." En déduiriez-vous que Betty veut que Fred n'écoute pas toutes les symphonies de Beethoven?

- "Betty veut que Fred écoute certains des albums de Renaud." En déduiriezvous que Betty veut que Fred n'écoute pas tous les albums de Renaud?

- "Betty veut que Fred écoute certains des opéras de Verdi." En déduiriez-vous 
que Betty veut que Fred n'écoute pas tous les opéras de Verdi?

- "Betty veut que Fred achète certaines des pièces de Racine." En déduiriez-vous que Betty veut que Fred n'achète pas toutes les pièces de Racine?

- "Betty veut que Fred achète certaines des symphonies de Beethoven." En déduiriez-vous que Betty veut que Fred n'achète pas toutes les symphonies de Beethoven?

- "Betty veut que Fred achète certains des albums de Renaud." En déduiriezvous que Betty veut que Fred n'achète pas tous les albums de Renaud?

- "Betty veut que Fred achète certains des Alien." En déduiriez-vous que Betty veut que Fred n'achète pas tous les Alien?

- "Betty veut que Fred achète certains des DVD de Harry Potter." En déduiriezvous que Betty veut que Fred n'achète pas tous les DVD de Harry Potter?

- "Betty veut que Fred achète certains des films de Tarantino." En déduiriezvous que Betty veut que Fred n'achète pas tous les films de Tarantino?

- "Betty veut que Fred achète certains des Harry Potter." En déduiriez-vous que Betty veut que Fred n'achète pas tous les Harry Potter?

- "Betty veut que Fred achète certains des James Bond." En déduiriez-vous que Betty veut que Fred n'achète pas tous les James Bond?

- "Betty veut que Fred achète certains des opéras de Verdi." En déduiriez-vous que Betty veut que Fred n'achète pas tous les opéras de Verdi?

- "Betty veut que Fred achète certains des romans de Daniel Pennac." En déduiriez-vous que Betty veut que Fred n'achète pas tous les romans de Daniel Pennac?

- "Betty veut que Fred achète certains des romans de Flaubert." En déduiriezvous que Betty veut que Fred n'achète pas tous les romans de Flaubert?

- "Betty veut que Fred lise certaines des pièces de Racine.” En déduiriez-vous que Betty veut que Fred ne lise pas toutes les pièces de Racine?

- "Betty veut que Fred lise certains des Harry Potter." En déduiriez-vous que Betty veut que Fred ne lise pas tous les Harry Potter?

- "Betty veut que Fred lise certains des poèmes des Fleurs du Mal." En déduiriez-vous que Betty veut que Fred ne lise pas tous les poèmes des Fleurs du Mal?

- "Betty veut que Fred lise certains des romans de Daniel Pennac." En déduiriezvous que Betty veut que Fred ne lise pas tous les romans de Daniel Pennac?

- "Betty veut que Fred loue certaines des saisons de Sex in the City." En déduiriez-vous que Betty veut que Fred ne loue pas toutes les saisons de Sex in the City?

- "Betty veut que Fred loue certaines des saisons de Sex in the City." En 
Background for "Embedded implicatures?!?"

déduiriez-vous que Betty veut que Fred ne loue pas toutes les saisons de Sex in the City?

- "Betty veut que Fred loue certains des Alien." En déduiriez-vous que Betty veut que Fred ne loue pas tous les Alien?

- "Betty veut que Fred loue certains des DVD de Harry Potter." En déduiriezvous que Betty veut que Fred ne loue pas tous les DVD de Harry Potter?

- "Betty veut que Fred loue certains des films de Tarantino." En déduiriez-vous que Betty veut que Fred ne loue pas tous les films de Tarantino?

- "Betty veut que Fred loue certains des James Bond.” En déduiriez-vous que Betty veut que Fred ne loue pas tous les James Bond?

- "Betty veut que Fred voie certains des Alien." En déduiriez-vous que Betty veut que Fred ne voie pas tous les Alien?

- "Betty veut que Fred voie certains des films de Tarantino." En déduiriez-vous que Betty veut que Fred ne voie pas tous les films de Tarantino?

- "Betty veut que Fred voie certains des Harry Potter." En déduiriez-vous que Betty veut que Fred ne voie pas tous les Harry Potter?

- "Betty veut que Fred voie certains des James Bond." En déduiriez-vous que Betty veut que Fred ne voie pas tous les James Bond?

\section{Control sentences}

"Fred a écouté certaines des chansons du dernier album de Madonna." En déduiriez-vous que Fred n'a pas écouté toutes les chansons du dernier album de Madonna?

- "Fred a écouté certaines des symphonies de Beethoven.” En déduiriez-vous que Fred n'a pas écouté toutes les symphonies de Beethoven?

- "Fred a écouté certains des albums de Renaud.” En déduiriez-vous que Fred n'a pas écouté tous les albums de Renaud?

- "Fred a écouté certains des opéras de Verdi." En déduiriez-vous que Fred n’a pas écouté tous les opéras de Verdi?

- "Fred a acheté certaines des pièces de Racine." En déduiriez-vous que Fred n'a pas acheté toutes les pièces de Racine?

- "Fred a acheté certaines des symphonies de Beethoven." En déduiriez-vous que Fred n'a pas acheté toutes les symphonies de Beethoven?

- "Fred a acheté certains des albums de Renaud." En déduiriez-vous que Fred n'a pas acheté tous les albums de Renaud?

- "Fred a acheté certains des Alien.” En déduiriez-vous que Fred n'a pas acheté tous les Alien? 
- "Fred a acheté certains des DVD de Harry Potter." En déduiriez-vous que Fred n'a pas acheté tous les DVD de Harry Potter?

- "Fred a acheté certains des films de Tarantino." En déduiriez-vous que Fred n'a pas acheté tous les films de Tarantino?

- "Fred a acheté certains des Harry Potter." En déduiriez-vous que Fred n’a pas acheté tous les Harry Potter?

- "Fred a acheté certains des James Bond." En déduiriez-vous que Fred n’a pas acheté tous les James Bond?

- "Fred a acheté certains des opéras de Verdi." En déduiriez-vous que Fred n’a pas acheté tous les opéras de Verdi?

- "Fred a acheté certains des romans de Daniel Pennac." En déduiriez-vous que Fred n'a pas acheté tous les romans de Daniel Pennac?

- "Fred a acheté certains des romans de Flaubert." En déduiriez-vous que Fred n'a pas acheté tous les romans de Flaubert?

- "Fred a loué certaines des saisons de Sex in the City." En déduiriez-vous que Fred n'a pas loué toutes les saisons de Sex in the City?

- "Fred a loué certains des Alien.” En déduiriez-vous que Fred n’a pas loué tous les Alien?

- "Fred a loué certains des DVD de Harry Potter." En déduiriez-vous que Fred n'a pas loué tous les DVD de Harry Potter?

- "Fred a loué certains des films de Tarantino." En déduiriez-vous que Fred n’a pas loué tous les films de Tarantino?

- "Fred a loué certains des James Bond." En déduiriez-vous que Fred n'a pas loué tous les James Bond?

- "Fred a lu certaines des pièces de Racine." En déduiriez-vous que Fred n’a pas lu toutes les pièces de Racine?

- "Fred a lu certains des Harry Potter." En déduiriez-vous que Fred n’a pas lu tous les Harry Potter?

- "Fred a lu certains des poèmes des Fleurs du Mal." En déduiriez-vous que Fred n'a pas lu tous les poèmes des Fleurs du Mal?

- "Fred a lu certains des romans de Daniel Pennac." En déduiriez-vous que Fred n'a pas lu tous les romans de Daniel Pennac?

- "Fred a lu certains des romans de Flaubert." En déduiriez-vous que Fred n'a pas lu tous les romans de Flaubert?

- "Fred a vu certaines des saisons de Sex in the City." En déduiriez-vous que Fred n'a pas vu toutes les saisons de Sex in the City?

- "Fred a vu certains des Alien." En déduiriez-vous que Fred n’a pas vu tous les Alien? 
Background for "Embedded implicatures?!?"

- "Fred a vu certains des films de Tarantino." En déduiriez-vous que Fred n’a pas vu tous les films de Tarantino?

- "Fred a vu certains des Harry Potter." En déduiriez-vous que Fred n’a pas vu tous les Harry Potter?

- "Fred a vu certains des James Bond." En déduiriez-vous que Fred n’a pas vu tous les James Bond?

\section{Complex sentences with deontic "must"}

"Il faut que Fred écoute certaines des chansons du dernier album de Madonna." En déduiriez-vous que Fred n'a pas le droit d'écouter toutes chansons du dernier album de Madonna?

- "Il faut que Fred écoute certaines des symphonies de Beethoven." En déduiriezvous que Fred n'a pas le droit d'écouter toutes les symphonies de Beethoven?

- "Il faut que Fred écoute certains des albums de Renaud." En déduiriez-vous que Fred n'a pas le droit d'écouter tous les albums de Renaud?

- "Il faut que Fred écoute certains des opéras de Verdi." En déduiriez-vous que Fred n'a pas le droit d'écouter tous les opéras de Verdi?

- "Il faut que Fred achète certaines des pièces de Racine." En déduiriez-vous que Fred n'a pas le droit d'acheter toutes les pièces de Racine?

- "Il faut que Fred achète certaines des symphonies de Beethoven." En déduiriezvous que Fred n'a pas le droit d'acheter toutes les symphonies de Beethoven?

- "Il faut que Fred achète certains des albums de Renaud." En déduiriez-vous que Fred n'a pas le droit d'acheter tous les albums de Renaud?

- "Il faut que Fred achète certains des Alien.” En déduiriez-vous que Fred n'a pas le droit d'acheter tous les Alien?

- "Il faut que Fred achète certains des DVD de Harry Potter." En déduiriez-vous que Fred n'a pas le droit d'acheter tous les DVD de Harry Potter?

- "Il faut que Fred achète certains des films de Tarantino." En déduiriez-vous que Fred n'a pas le droit d'acheter tous les films de Tarantino?

- "Il faut que Fred achète certains des Harry Potter." En déduiriez-vous que Fred n'a pas le droit d'acheter tous les Harry Potter?

- "Il faut que Fred achète certains des James Bond." En déduiriez-vous que Fred n'a pas le droit d'acheter tous les James Bond?

- "Il faut que Fred achète certains des opéras de Verdi." En déduiriez-vous que Fred n'a pas le droit d'acheter tous les opéras de Verdi?

- "Il faut que Fred achète certains des romans de Daniel Pennac." En déduiriezvous que Fred n'a pas le droit d'acheter tous les romans de Daniel Pennac? 
- "Il faut que Fred achète certains des romans de Flaubert." En déduiriez-vous que Fred n'a pas le droit d'acheter tous les romans de Flaubert?

- "Il faut que Fred lise certaines des pièces de Racine." En déduiriez-vous que Fred n'a pas le droit de lire toutes les pièces de Racine?

- "Il faut que Fred lise certains des Harry Potter." En déduiriez-vous que Fred n'a pas le droit de lire tous les Harry Potter?

- "Il faut que Fred lise certains des poèmes des Fleurs du Mal." En déduiriezvous que Fred n'a pas le droit de lire tous les poèmes des Fleurs du Mal?

- "Il faut que Fred lise certains des romans de Daniel Pennac." En déduiriezvous que Fred n'a pas le droit de lire tous les romans de Daniel Pennac?

- "Il faut que Fred lise certains des romans de Flaubert." En déduiriez-vous que Fred n'a pas le droit de lire tous les romans de Flaubert?

- "Il faut que Fred loue certaines des saisons de Sex in the City." En déduiriezvous que Fred n'a pas le droit de louer toutes les saisons de Sex in the City?

- "Il faut que Fred loue certains des Alien." En déduiriez-vous que Fred n’a pas le droit de louer tous les Alien?

- "Il faut que Fred loue certains des DVD de Harry Potter." En déduiriez-vous que Fred n'a pas le droit de louer tous les DVD de Harry Potter?

- "Il faut que Fred loue certains des films de Tarantino." En déduiriez-vous que Fred n'a pas le droit de louer tous les films de Tarantino?

- "Il faut que Fred loue certains des James Bond." En déduiriez-vous que Fred n'a pas le droit de louer tous les James Bond?

- "Il faut que Fred voie certaines des saisons de Sex in the City." En déduiriezvous que Fred n'a pas le droit de voir toutes les saisons de Sex in the City?

- "Il faut que Fred voie certains des Alien." En déduiriez-vous que Fred n’a pas le droit de voire tous les Alien?

- "Il faut que Fred voie certains des films de Tarantino." En déduiriez-vous que Fred n'a pas le droit de voir tous les films de Tarantino?

- "Il faut que Fred voie certains des Harry Potter." En déduiriez-vous que Fred n'a pas le droit de voire tous les Harry Potter?

- "Il faut que Fred voie certains des James Bond." En déduiriez-vous que Fred n'a pas le droit de voire tous les James Bond?

\section{Complex sentences with "all"}

"Tous les étudiants ont écouté certaines des chansons du dernier album de Madonna." En déduiriez-vous qu'aucun des étudiants n'a écouté toutes les 
Background for "Embedded implicatures?!?"

chansons du dernier album de Madonna?

- "Tous les étudiants ont écouté certaines des opéras de Verdi." En déduiriezvous qu'aucun des étudiants n’a écouté tous les opéras de Verdi?

- "Tous les étudiants ont écouté certaines des symphonies de Beethoven." En déduiriez-vous qu'aucun des étudiants n’a écouté toutes les symphonies de Beethoven?

- "Tous les étudiants ont écouté certains des albums de Renaud." En déduiriezvous qu'aucun des étudiants n'a écouté tous les albums de Renaud?

- "Tous les étudiants ont acheté certaines des opéras de Verdi." En déduiriezvous qu'aucun des étudiants n’a acheté tous les opéras de Verdi?

- "Tous les étudiants ont acheté certaines des pièces de Racine." En déduiriezvous qu'aucun des étudiants n'a acheté toutes les pièces de Racine?

- "Tous les étudiants ont acheté certaines des symphonies de Beethoven." En déduiriez-vous qu'aucun des étudiants n'a acheté toutes les symphonies de Beethoven?

- "Tous les étudiants ont acheté certains des albums de Renaud." En déduiriezvous qu'aucun des étudiants n'a acheté tous les albums de Renaud?

- "Tous les étudiants ont acheté certains des Alien.” En déduiriez-vous qu'aucun des étudiants n'a acheté tous les Alien?

- "Tous les étudiants ont acheté certains des DVD de Harry Potter." En déduiriez-vous qu'aucun des étudiants n'a acheté tous les DVD de Harry Potter?

- “Tous les étudiants ont acheté certains des films de Tarantino." En déduiriezvous qu'aucun des étudiants n'a acheté tous les films de Tarantino?

- "Tous les étudiants ont acheté certains des Harry Potter." En déduiriez-vous qu'aucun des étudiants n'a acheté tous les Harry Potter?

- "Tous les étudiants ont acheté certains des James Bond." En déduiriez-vous qu'aucun des étudiants n'a acheté tous les James Bond?

- "Tous les étudiants ont acheté certains des romans de Daniel Pennac." En déduiriez-vous qu'aucun des étudiants n'a acheté tous les romans de Daniel Pennac?

- "Tous les étudiants ont acheté certains des romans de Flaubert." En déduiriezvous qu'aucun des étudiants n'a acheté tous les romans de Flaubert?

- “Tous les étudiants ont aloué certains des James Bond.” En déduiriez-vous qu'aucun des étudiants n'a loué tous les James Bond?

- "Tous les étudiants ont loué certaines des saisons de Sex in the City." En déduiriez-vous qu'aucun des étudiants n'a loué toutes les saisons de Sex in the City? 
- “Tous les étudiants ont loué certains des Alien.” En déduiriez-vous qu'aucun des étudiants n'a loué tous les Alien?

- "Tous les étudiants ont loué certains des DVD de Harry Potter." En déduiriezvous qu'aucun des étudiants n'a loué tous les DVD de Harry Potter?

- "Tous les étudiants ont loué certains des films de Tarantino." En déduiriezvous qu'aucun des étudiants n'a loué tous les films de Tarantino?

- "Tous les étudiants ont lu certaines des pièces de Racine." En déduiriez-vous qu'aucun des étudiants n'a lu toutes les pièces de Racine?

- "Tous les étudiants ont lu certains des Harry Potter." En déduiriez-vous qu'aucun des étudiants n'a lu tous les Harry Potter?

- "Tous les étudiants ont lu certains des poèmes des Fleurs du Mal." En déduiriez-vous qu'aucun des étudiants n'a lu tous les poèmes des Fleurs du Mal?

- "Tous les étudiants ont lu certains des romans de Daniel Pennac." En déduiriez-vous qu'aucun des étudiants n'a lu tous les romans de Daniel Pennac?

- "Tous les étudiants ont lu certains des romans de Flaubert." En déduiriezvous qu'aucun des étudiants n'a lu tous les romans de Flaubert?

- "Tous les étudiants ont vu certaines des saisons de Sex in the City." En déduiriez-vous qu'aucun des étudiants n'a vu toutes les saisons de Sex in the City?

- “Tous les étudiants ont vu certains des Alien." En déduiriez-vous qu'aucun des étudiants n'a vu tous les Alien?

- "Tous les étudiants ont vu certains des films de Tarantino." En déduiriez-vous qu'aucun des étudiants n'a vu tous les films de Tarantino?

- "Tous les étudiants ont vu certains des Harry Potter." En déduiriez-vous qu'aucun des étudiants n'a vu tous les Harry Potter?

- "Tous les étudiants ont vu certains des James Bond.” En déduiriez-vous qu'aucun des étudiants n’a vu tous les James Bond?

\section{Experiment 2: Instructions}

We give here the instructions for those partipants who saw the verification task first. 
Background for "Embedded implicatures?!?"

\section{Verification task}

Dit experiment bestaat uit twee delen. Hier volgen de instructies voor het eerste deel. Op elk van de volgende bladzijden zie je een bewering en een afbeelding van twee vakken met letters erin. De vraag is of de zin waar is in de gegeven situatie. Bijvoorbeeld, in de volgende situatie:

\section{B B A C A A C}

is de zin "Het rechtervak bevat meer dan één A" waar en je kruist dus "waar" aan.

Werk de items één voor één door en blader niet vooruit of terug. Je hoeft je niet te haasten, maar anderzijds is het ook niet de bedoeling om al te lang na te denken. Geef gewoon het antwoord dat het eerste bij je opkomt.

\section{Inference task}

In het tweede gedeelte van dit experiment bevat iedere bladzijde twee zinnen en het is de bedoeling om te bepalen of de tweede zin uit de eerste zin mag worden afgeleid. Bijvoorbeeld, als iemand zegt:

"Het linkervak bevat twee A's en twee B's."

zou je hieruit kunnen afleiden dat het linkervak volgens de spreker meer dan drie letters bevat? In dit geval kruis je dus "ja” aan.

Werk de items één voor één door en blader niet vooruit of terug. Je hoeft je niet te haasten, maar anderzijds is het ook niet de bedoeling om al te lang na te denken. Geef gewoon het antwoord dat het eerste bij je opkomt.

\section{Experiment 3: Instructions}

\section{Verification task}

Op elke bladzijde zie je een zin met daarboven een afbeelding bestaande uit vierkanten en cirkels die door lijnen met elkaar verbonden zijn. Het gaat erom te bepalen of de zin waar is in de afgebeelde situatie.

Werk de items één voor één door en blader niet vooruit of terug. Het gaat er in dit experiment niet om hoe snel je bent. Je hoeft je dus niet te haasten, 
maar anderzijds is het ook niet de bedoeling om al te lang na te denken. Geef gewoon het antwoord dat het eerste bij je opkomt.

\section{Inference task}

Elke bladzijde ziet er ongeveer uit als volgt:

Betty zegt:
Er zijn precies twee cirkels die met enkele vierkanten
verbonden zijn
Zou je hieruit kunnen opmaken dat volgens Betty:
er precies twee vierkanten zijn die met enkele cirkels
verbonden zijn
$\square$ ja
$\square$ nee

"Betty" is een willekeurige persoon.

Werk de items één voor één door en blader niet vooruit of terug. Het gaat er in dit experiment niet om hoe snel je bent. Je hoeft je dus niet te haasten, maar anderzijds is het ook niet de bedoeling om al te lang na te denken. Geef gewoon het antwoord dat het eerste bij je opkomt. 
Background for "Embedded implicatures?!?"

\section{Experiment 3: Materials}

\section{Verification task}

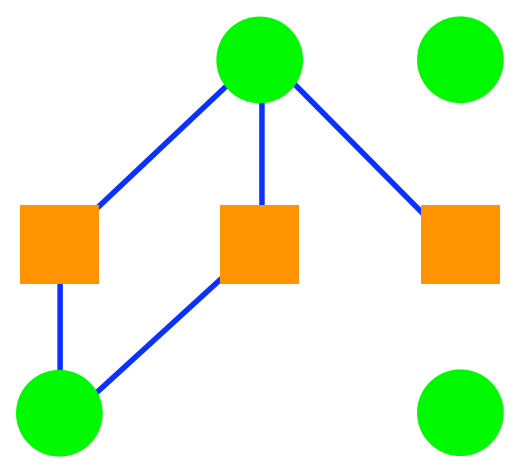

Er zijn precies twee cirkels die met enkele vierkanten verbonden zijn.

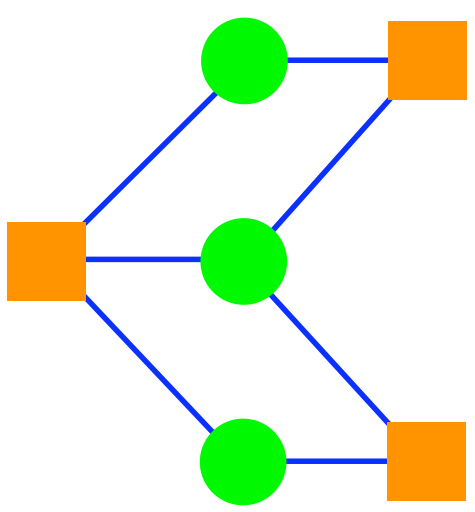

Alle vierkanten zijn met enkele cirkels verbonden. 
Bart Geurts \& Nausicaa Pouscoulous

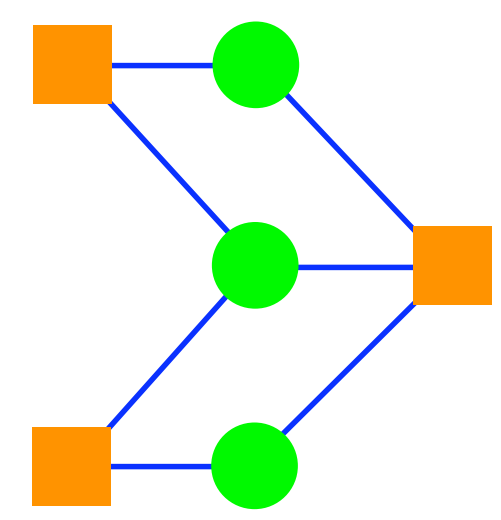

Niet alle vierkanten zijn met enkele cirkels verbonden.

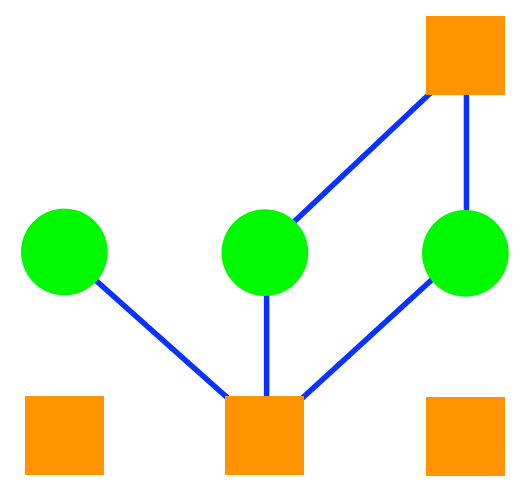

Er is meer dan één vierkant dat met enkele cirkels verbonden is. 
Background for "Embedded implicatures?!?"

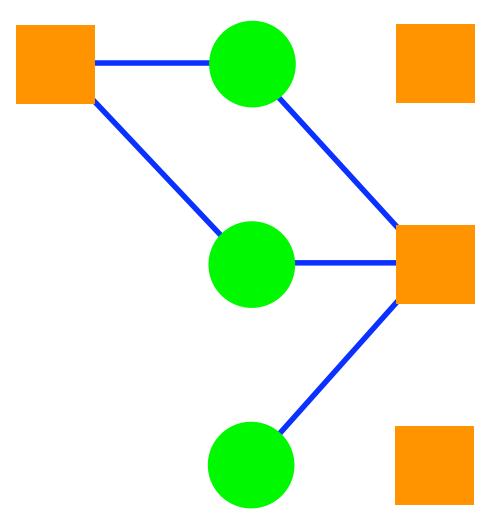

Er is niet meer dan één vierkant dat met enkele cirkels verbonden is.

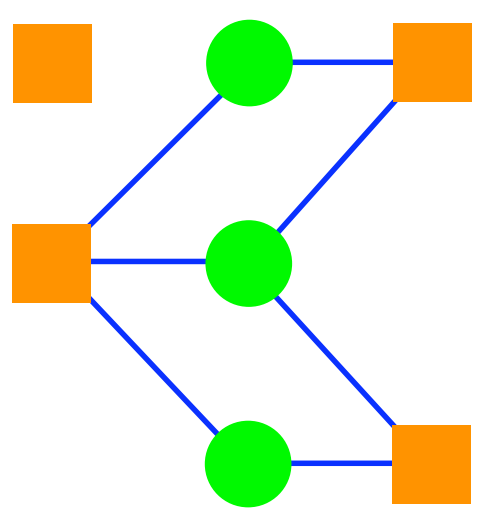

Er zijn precies twee vierkanten die met enkele cirkels verbonden zijn.

\section{Inference task}

- Betty zegt: "Er is meer dan één vierkant dat met enkele cirkels verbonden is.” Zou je hieruit kunnen opmaken dat ze bedoelt dat er is meer dan één vierkant is dat met enkele maar niet alle cirkels verbonden is.

- Betty zegt: “Alle cirkels zijn met enkele vierkanten verbonden.” Zou je hieruit kunnen opmaken dat ze bedoelt dat alle cirkels met enkele maar niet alle vierkanten verbonden zijn. 
- Betty zegt: "Er zijn precies twee cirkels die met enkele vierkanten verbonden zijn.” Zou je hieruit kunnen opmaken dat ze bedoelt dat er precies twee cirkels zijn die met enkele maar niet alle vierkanten verbonden zijn.

- Betty zegt: "Er is niet meer dan één vierkant dat met enkele cirkels verbonden is." Zou je hieruit kunnen opmaken dat ze bedoelt dat er niet meer dan één vierkant is dat met enkele maar niet alle cirkels verbonden is.

- Betty zegt: "Niet alle cirkels zijn met enkele vierkanten verbonden." Zou je hieruit kunnen opmaken dat ze bedoelt dat niet alle cirkels met enkele maar niet alle vierkanten verbonden zijn.

\section{Experiment 4: Materials}

\section{Scalar inferences}

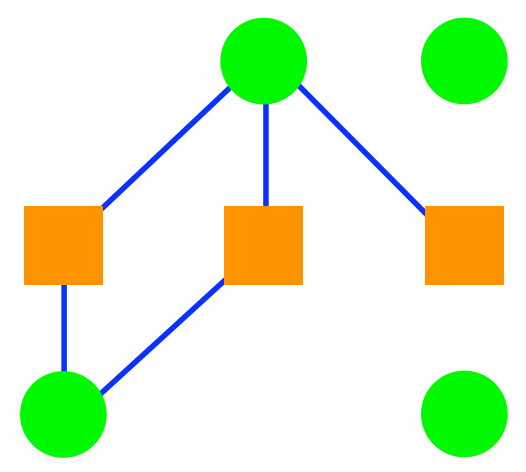

There are exactly two circles that are connected with some of the squares. 
Background for "Embedded implicatures?!?"

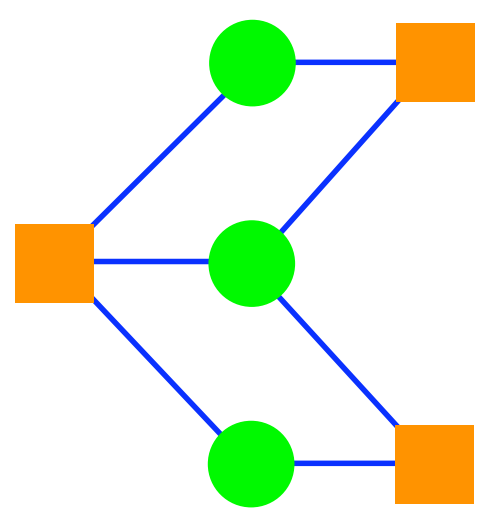

All the squares are connected with some of the circles.

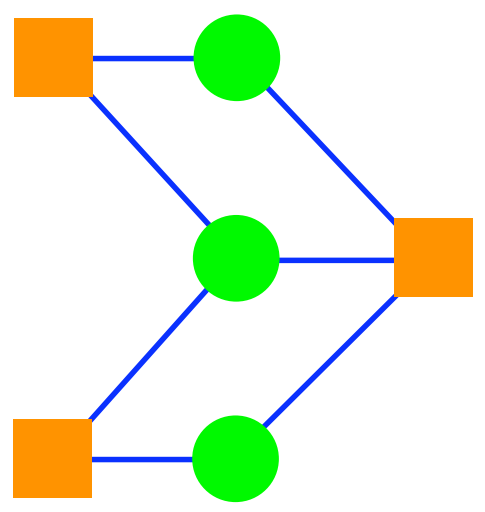

Not all the squares are connected with some of the circles. 
Bart Geurts \& Nausicaa Pouscoulous

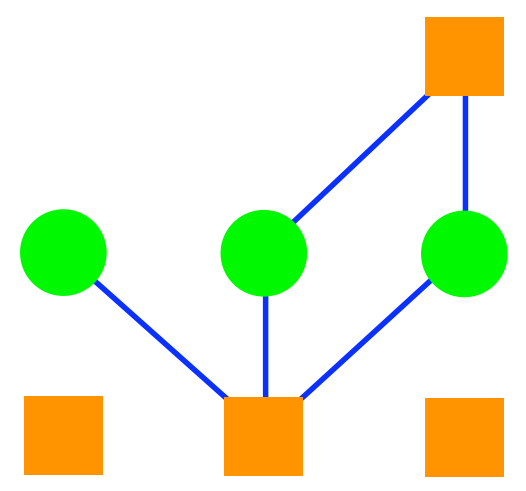

There is more than one square that is connected with some of the circles.

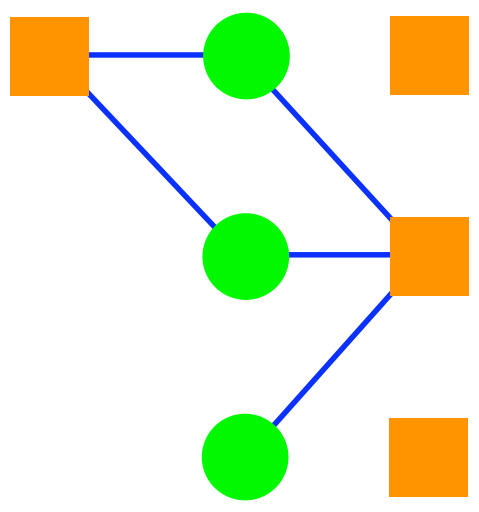

There isn't more than one square that is connected with some of the circles. 
Background for "Embedded implicatures?!?"

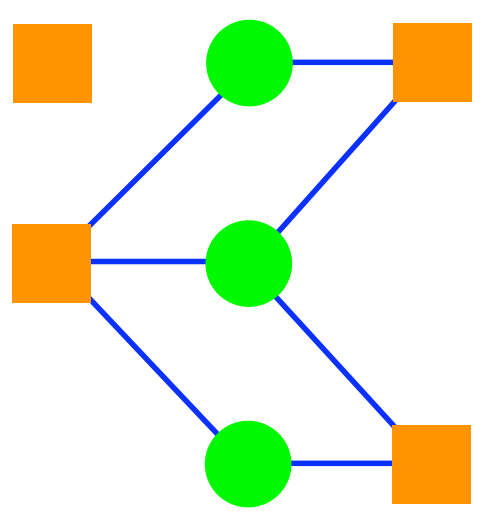

There are exactly two squares that are connected with some of the circles.

\section{Ambiguous controls}

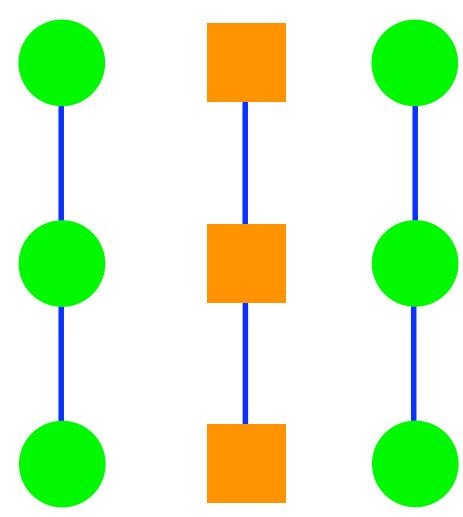

The circles and the squares are connected with each other. 
Bart Geurts \& Nausicaa Pouscoulous

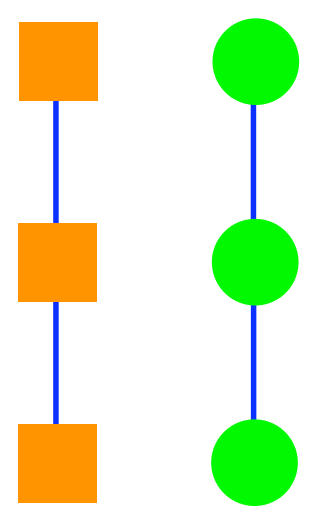

The green and the orange figures are connected with each other.

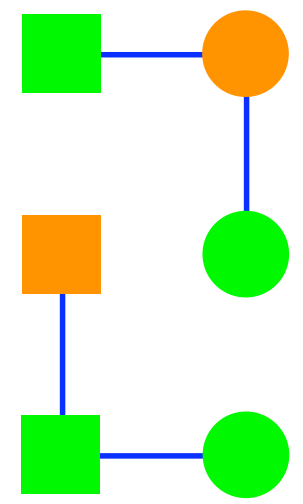

All the figures are orange and green. 
Background for "Embedded implicatures?!?"

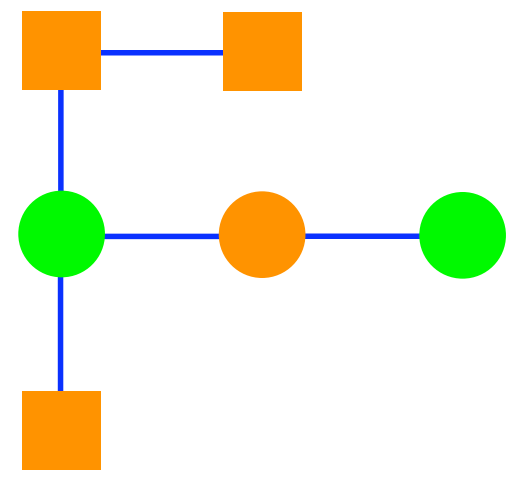

There are green circles and squares.
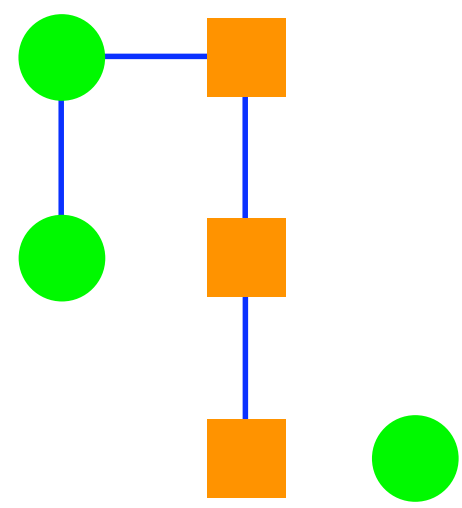

The circles and the squares have the same colour. 